\title{
LHeC and eRHIC
}

\author{
Vladimir N Litvinenko ${ }^{1}$ on behalf of eRHIC and LHeC teams ${ }^{2}$ \\ Center for Accelerator Science and Education \\ Brookhaven National Laboratory, Upton, NY, USA \\ Stony Brook University, Stony Brook, NY, USA \\ BNL, P.O.Box 5000, MS-911, Upton, NY 11973-5000, USA \\ E-mail: vI $a b n I . g \circ \mathrm{V}$
}

This paper is focused on possible designs and predicted performances of two proposed highenergy, high-luminosity electron-hadron colliders: eRHIC at Brookhaven National Laboratory (BNL, Upton, NY, USA) and LHeC at Organisation Européenne pour la Recherche Nucléaire (CERN, Geneve, Switzerland). The Relativistic Heavy Ion Collider (RHIC, BNL) and the Large Hadron Collider (LHC, CERN) are designed as versatile colliders. RHIC is colliding various species of hadrons staring from polarized protons to un-polarized heavy ions (such as fully stripped $\mathrm{Au}$ (gold) ions) in various combinations: polarized p-p, d-Au, $\mathrm{Cu}-\mathrm{Cu}, \mathrm{Au}-\mathrm{Au}$. Maximum energy in RHIC is $250 \mathrm{GeV}$ (per beam) for polarized protons and $100 \mathrm{GeV} / \mathrm{n}$ for heavy ions. There is planed expansion of the variety of species to include polarized $\mathrm{He}^{3}$ and unpolarized fully stripped $\mathrm{U}$ (uranium). $\mathrm{LHeC}$ is designed to collide both un-polarized protons with energy up to $7 \mathrm{TeV}$ per beam and fully stripped $\mathrm{Pb}$ (lead) ions with energy up to $3 \mathrm{TeV} / \mathrm{n}$.

Both eRHIC and LHeC plan to add polarized electrons (or/and positrons) to the list of colliding species in these versatile hadron colliders. In eRHIC 10-20 GeV electrons would collide with hadrons circulating in RHIC. In LHeC 50-150 GeV polarized leptons will collided with LHC's hadron beams. Both colliders plan to operate in electron-proton (in RHIC case protons are polarized as well) and electron-ion collider modes. eRHIC and LHeC colliders are complimentary both in the energy reach and in their physics goals. I will discuss in this paper possible choices of the accelerator technology for the electron part of the collider for both eRHIC and $\mathrm{LHeC}$, and will present predicted performance for the colliders. In addition, possible staging scenarios for these colliders will be discussed.

The 2009 Europhysics Conference on High Energy Physics,

Krakow, Poland

July 16-22, 2009

\footnotetext{
$1 \quad$ Vladimir N Litvinenko, vl@bnl.gov

${ }^{2}$ eRHIC team: M.Bai, J.Beebe-Wang, I.Ben-Zvi, M.Blaskiewicz, A. Burrill, R.Calaga, X.Chang, A.Drees, A.V.Fedotov, H.Hahn, L.Hammons, Y.Hao, H.Huang, A.Jain, D.Kayran, J.Kewisch, A.Lucio, G.McIntyre, B.Parker, E.Pozdeyev, V.Ptitsyn, T.Roser, S.Tepikian, D.Trbojevic, N.Tsoupas, J.Tuozzolo, G.Wang, S.Webb, A.Zelensky (BNL), E.Tsentalovich (MIT)

LHeC team: F.Zimmermann, F.Bordry, H.-H.Braun, O.S.Brüning, H.Burkhardt, A.Eide, A.de Roeck, R.Garoby, B.Holzer, J.M.Jowett, T.Linnecar, K.-H.Mess, J.Osborne, L.Rinolfi, D.Schulte, R.Tomas, J.Tückmantel, A.Vivoli (CERN), S.Chattopadhyay, J.Dainton (Cockcroft Inst., UK), M.Klein (U.Liverpool, UK), T.Omori, J.Urakawa (KEK, Japan, F.Willeke, (BNL, USA), A.K.Ciftci (Ankara U.), H.Aksakal, U.Nigde, S.Sultansoy (TOBB ETU, Turkey)
} 


\section{Introduction}

Great successes achieved by first - and up-to-date the only - electron hadron collider HERA (DESY, Germany) [1,2] stimulated intense interest in both the accelerator and the high energy and nuclear physics communities. Abilities of electron-hadron collider to provide information on nucleon structure complimentary to that obtained in hadron and lepton colliders and very high precision of its data were and are behind this interest. Many of existing and proposed hadron colliders had consider or are considering an option of adding a lepton accelerator and to operate them also as electron hadron-colliders. Presently there are four groups developing such proposals. Three groups at BNL, CERN and GSI (Helmholtz Centre for Heavy Ion Research, Germany) plan to add lepton accelerator to the existing (eRHIC at BNL and LHeC at CERN) or future (ENC at FAIR, GSI) [3] hadron facilities. Fourth group at Thomas Jefferson National Accelerator Facility (TJNAF, USA) suggest to existing CEBAF facility as an injector of polarized electron into ELIC -collider with new hadron complex and new lepton and hadron rings [4]. Two later proposals, the ENC and the ELIC, were not topic of my presentation at the conference and, therefore, are not discussed in this paper.

This paper is focused on eRHIC and LHeC designs, their common feature and their differences.

\section{2. eRHIC}

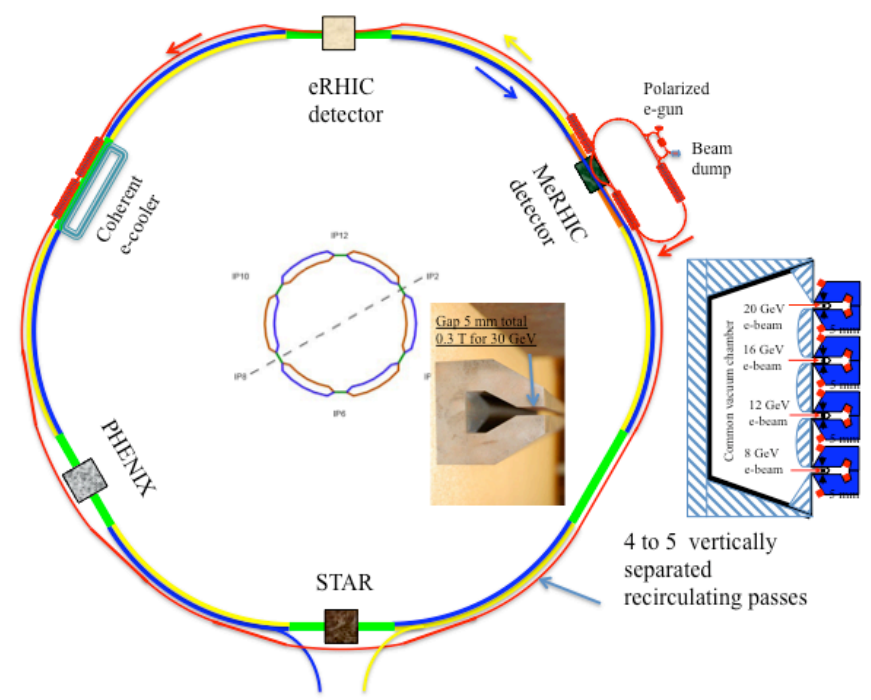

Fig. 1. Possible layout of eRHIC with $20 \mathrm{GeV}$ energy-recovery linac colliding with RHIC beam at 12 o'clock interaction region.

As shown in Fig.1, RHIC is comprised from two $3.8 \mathrm{~km}$ long super-conducting rings (called Blue and Yellow rings), which cross each other six times at 2, 4, 6, 8, 10 and 12 o'clock interaction regions. Starting from 1999s a discussion of possible RHIC upgrade by adding a lepton accelerator had been initiated [5]. These discussions of eRHIC had been followed by workshops on possible physics with such electron-ion collider (EIC) [6] and EIC collaboration 
had been established [7]. Since first workshop in 1999 at Indiana University, there were twenty meetings on EIC physics with both eRHIC and ELIC colliders under consideration.

In March 2004, a collaboration of accelerator physics from BNL, MIT-Bates (MA, USA), BINP (Novosibirsk, Russia) and DESY developed and published $0^{\text {th }}$ Order Design Report on eRHIC [8]. The report considered two options for eRHIC: a ring-ring and a linacring. The ring-ring option was considered at that time as the main option for eRHIC and was based on 5-to- $10 \mathrm{GeV}$ stand-alone electron storage ring with it circumference being 1/3 of that of RHIC. The polarized electrons, generated by a polarized Photoinjector gun, would be accelerated and injected in the ring by a full energy recirculating linac. The case of the linacring eRHIC version based on a multi-pass Energy Recovery Linac (ERL) was also studied as a possible back-up option with potential for higher luminosity. The report was followed up by rather detailed cost estimate for the ring-ring option, and a less rigorous top-down cost estimate for the linac-ring option. The costs for both options were very similar. At that time the main argument for choosing the ring-ring as the baseline option was that the ring-ring is based on standard technology, while ERL was new developing technology. The linac-ring discussed at that time would also require a polarized electron source with $450 \mathrm{~mA}$ average current, which was two orders of magnitude above the demonstrated level.

Table 1. Parameters of eRHIC electron-hadron collider

\begin{tabular}{|l|c|c|c|c|}
\hline & \multicolumn{2}{|c|}{ MeRHIC } & \multicolumn{2}{c|}{ eRHIC* } \\
\cline { 2 - 5 } & $\mathrm{p}(\mathrm{A})$ & $\mathrm{e}$ & $\mathrm{p}(\mathrm{A})$ & $\mathrm{e}$ \\
\hline Energy, GeV & $250(100)$ & 4 & $325(125)$ & 20 \\
\hline Number of bunches & 111 & & 166 & \\
\hline Bunch intensity $(\mathrm{u}), 10^{11}$ & 2.0 & 0.31 & $2.0(3)$ & 0.24 \\
\hline Bunch charge, $\mathrm{nC}$ & 32 & 5 & 32 & 4 \\
\hline Beam current, $\mathrm{mA}$ & 320 & 50 & 420 & 50 \\
\hline $\begin{array}{l}\text { Normalized emittance, } \mu \mathrm{m}, \\
95 \% \text { for hadrons } / \mathrm{rms} \text { for e }\end{array}$ & 15 & 73 & 1.2 & 25 \\
\hline Polarization, $\%$ & 70 & 80 & 70 & 80 \\
\hline rms bunch length, cm & 20 & 0.2 & 4.9 & 0.2 \\
\hline$\beta^{*}$, cm & 50 & 50 & 25 & 25 \\
\hline Luminosity, $\mathrm{c} 10^{33}, \mathrm{~cm}^{-2} \mathrm{~s}^{-1}$ & & $0.1-1 *$ & & $2.8^{*}$ \\
\hline
\end{tabular}

* Assumes cooling of the hadron beam

Detailed studies of both options in the following years clearly demonstrated that linac-ring version of eRHIC can provide significantly (up to order of the magnitude) luminosity compared with the ring-ring version $[9,10]$. At any given set of beam parameters and any given design of interaction region, the linac-ring collider out-performs its ring-ring contra-part by a significant margin. In the ring-ring collider, the luminosity is limited by allowable beam-beam tune fro both the hadron and the electron beam. Use of ERL (or linac) as electron accelerator 
dramatically changes this picture: electron beam is used only once for the collision(s) and is allowed be strongly disrupted by colliding electron beam [11]. The only condition that this beam can be decelerated in the ERL and damped is satisfied in all practical case under consideration. Furthermore, our studies showed that linac-ring version of eRHIC can take full advantage of electron cooling and reduce electron beam current requirements to $50 \mathrm{~mA}$, which can be generated by a multi-cathode Gatling gun [12]. Operating at low current allowed us to consider higher energy operation of eRHIC with top electron energy of 20 or even $30 \mathrm{GeV}$. In 2007, we selected the ERL based eRHIC as a baseline.

Being a linac-based eRHIC has a very straightforward staging strategy [13]. One of the latest options of the eRHIC layout is shown in Fig.1, while Table 1 list eRHIC parameters fro two main stages. A detailed design of the first stage of eRHIC - MeRHIC - had been developed in significant details, including the cost estimate to the accelerator. The first version of MeRHIC technical design planned to be published on web in November, 2009.

\section{LHeC}
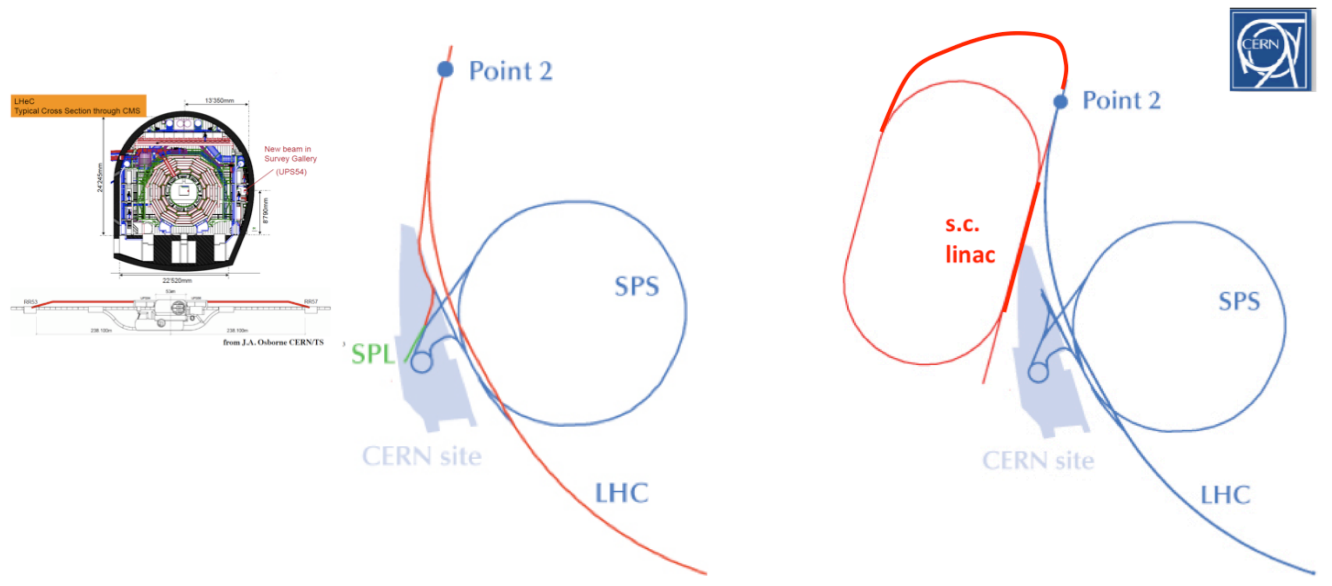

Fig. 2. Sketches of two possible layouts of LHeC: ring-ring option is shown on the left, and linac-ring option is shown on the right. Electron ring would be located inside the LHC tunnel with necessary bypasses around LHC detectors. The linac (or

Discussions of adding leptons to the LHC started as early as those at RHIC and the first paper on $\mathrm{LHeC}$ was published in 1997 [14]. First LHeC workshop was held in 2008 [15-16], with a number of dedicated workshops following it. At present, two main options are considered for LHeC: a ring-ring and a linac-ring [17-22] (see Fig.2). The linac-ring option has also two possible scenarios [17] - a pulsed linac without energy recovery and an ERL.

Adding 50 to $150 \mathrm{GeV}$ electron beam to the $\mathrm{LHeC}$ has its unique complications, which are different from the most existing colliders and also from eRHIC. Synchrotron radiation of such beams can consume significant part of its energy at one pass around the ring and operating such machine can be very power consuming.

A comparative study, with assumption that the AC plug power is set at $100 \mathrm{MW}$ level, was conducted by $\mathrm{LHeC}$ team. The resulting luminosity predictions are shown in Fig. 3. 


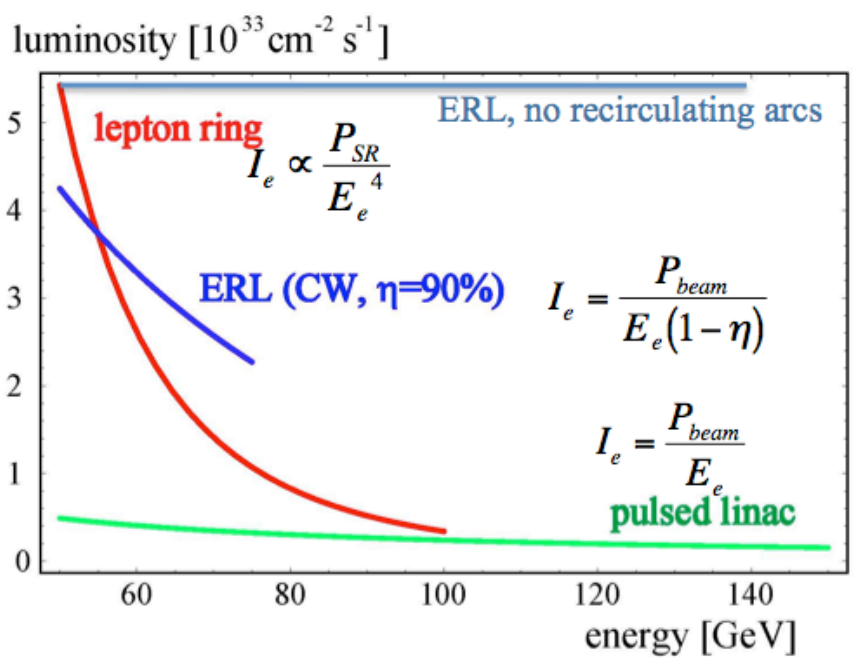

Fig. 3. LHeC luminosity attainable with a lepton ring, a pulsed linac and an energy recovery linac. The assumption is that the AC plug power is about $100 \mathrm{MW}$ for all three options. The horizontal axis is the electron (lepton) energy in $\mathrm{GeV}$.

In the case of the lepton ring, the synchrotron radiation, which growth at the power four of the beam energy, is the limiting factor for the lepton beam current and, therefore, for the luminosity. This limit the ring-ring luminosity below $10^{33}$ level after about $70 \mathrm{GeV}$ electron energy.

In the case of a pulsed linac, the allowable electron beam current is inversely proportional to its energy. This dependence is less dramatic than that of ring-ring case, and this option may have higher luminosity at energies above $100 \mathrm{GeV}$.

The most attractive option for linac-ring case is an ERL, where most of the e-beam energy is recovered. But in contrast with eRHIC, in $\mathrm{LHeC}$ case turning electron beam around the LHC tunnel will generate as much power loss for synchrotron radiation as in the ring. Therefore, ERL option with recirculating arcs is attractive only at modest energies.

At present time both concept are pursued as potentially viable candidates for LHeC. In all cases presently under consideration, the electron beam intensity is well below the level allowed by the beam-beam tune shift of the hadron beam in the LHC. In other words, LHeC luminosity is not limited by the beam-beam effects and has a potential for an increase.

Energy recovery linac without recirculating arcs (example described in [23]) can be used at any energy without loss of luminosity. Unfortunately the cost of such ERL can be high and this concept is not presently under consideration by $\mathrm{LHeC}$ team.

\section{Conclusions}

Two lepton-hadron colliders, eRHIC and $\mathrm{LHeC}$, will cover rather different energy ranges: eRHIC c.m. energy range would be $15-200 \mathrm{GeV}$, while $\mathrm{LHeC}$ would cover $0.5-2 \mathrm{TeV}$. Both collider designs are based on the beam parameters either achieved or predicted for the hadron 
part of the collider. Both promise to deliver very high average luminosity in $10^{33} \mathrm{~cm}^{-2} \mathrm{sec}^{-1}$ range, with some potential of upgrades.

\section{References}

[1] "Deep inelastic Compton scattering at the ep collider HERA", A.C.Bawa et al., DESY, Hamburg (Germany), 1990, http://hdl.handle.net/10068/275726

[2] "Collider physics at HERA", M. Klein and R. Yoshida, Progress in Particle and Nuclear Physics, Volume 61, Issue 2, October 2008, Pages 343-393

[3] A Concept for a Polarized Electron-Nucleon Collider at the HESR of the FAIR Project A.Lehrach et al., Proceedings of 2009 Particle Accelerator Conference, Vancouver, Canada, May 4-8, 2009

[4] Recent Progress on Design Studies of High-Luminosity Ring-Ring Electron-Ion Collider at CEBAF, Y. Zhang et al., Proceedings of 2009 Particle Accelerator Conference, Vancouver, Canada, May 4-8, 2009

[5] I. Ben-Zvi, J. Kewisch, J. Murphy and S. Peggs, "Accelerator Physics Issues in eRHIC," Nuclear Instruments and Methods in Physics Research A463, 94 (2001), C-A/AP/14

[6] "The Electron Ion Collider", A white paper summarizing the scientific opportunities and the preliminary detector and accelerator design options, February 2002, http://www.phenix.bnl.gov/WWW/publish/abhay/Home_of_EIC/Whitepaper/Final/

[7] The Electron-Ion Collider Collaboration, http://web.mit.edu/eicc/

[8] eRHIC $-0^{\text {th }}$ Order Design Report, Editors: M. Farkhondeh and V.Ptitsyn, BNL, 2004, http://www.bnl.gov/cad/eRHIC/eRHIC ZDR.asp

[9] V.N. Litvinenko et al., Proc. of 2005 Particle Accelerator Conference, May 2005, Knoxville, TN, 276,8 http://cern.ch/AccelConf/p05/PAPERS/TPPP043.PDF

[10] V. Ptitsyn et al., Proceedings of EPAC 2006, Edinburgh, Scotland, June 26-30 2006, http://accelconf.web.cern.ch/AccelConf/e06/PAPERS/MOPLS058.PDF

[11] Beam-Beam Interaction Study of Medium Energy eRHIC, Y.Hao, V.N. Litvinenko, V. Ptitsyn, Proceedings of 2009 Particle Accelerator Conference, Vancouver, Canada, May 48,2009 , and references there in

[12] V.N.Litvinenko, unpublished, 2001

[13] MeRHIC -- Staging Approach to eRHIC, V.Ptitsyn et al., Proceedings of 2009 Particle Accelerator Conference, Vancouver, Canada, May 4-8, 2009

[14] E. Keil, "LHC ep option", LHC-Project-Report-093, CERN Geneva 1997

[15] First ECFA-CERN LHeC Workshop, Divonne, Sept. 2008.

[16] LHeC web site: http://www.lhec.org.uk

[17] The Large Hadron-Electron Collider (LHeC) at the LHC, F. Zimmermann et all, Proceedings of 2009 Particle Accelerator Conference, Vancouver, Canada, May 4-8, 2009

[18] H. Burkhardt, "Ring-Ring Layout \& Bypass Design," First ECFA-CERN LHeC Workshop, Divonne, Sept. 2008

[19] F. Willeke et al, Proc. EPAC'08 Genoa, p. 2638 (2008)

[20] F. Zimmermann et al, Proc. EPAC'08 Genoa, p. 2847(2008)

[21] B. Holzer, DIS09, Madrid 2009, to be published

[22] M. Klein et al, CERN Courier, April '09, p. 22 (2009)

[23] V.N.Litvinenko, "LHeC with $\sim 100 \%$ energy recovery linac", $2^{\text {nd }}$ LHeC workshop, Divonne, Sept.2009, http://indico.cern.ch/sessionDisplay.py?sessionId=15\&slotId=0\&confId=59304\#2009-0902 